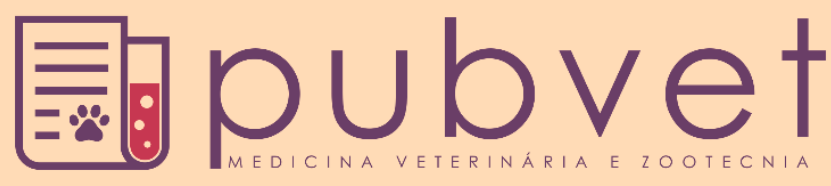

https://doi.org/10.31533/pubvet.v14n12a710.1-8

\title{
Persistência do ducto arterioso: Relato de caso
}

\author{
Fabiana Sperb Volkweis ${ }^{190}$, Gabriela Gonçalves Bowen $^{2 *}$, Guilherme Kanciukaitis Tognoli ${ }^{\sqrt{9}}$, \\ Josiane de Almeida Sales ${ }^{4} 9$
}

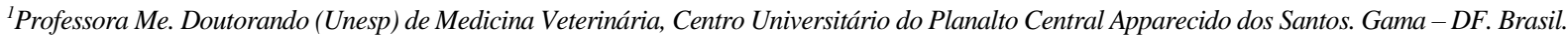
${ }^{2}$ Graduando em Medicina Veterinária, Centro Universitário do Planalto Central Apparecido dos Santos. Gama-DF. Brasil.

${ }^{3}$ Professor MSc, de Medicina Veterinária, Centro Universitário do Planalto Central Apparecido dos Santos. Gama-DF. Brasil.

${ }^{4}$ Médica Veterinária, Hospital Veterinário do Centro Universitário do Planalto Central Apparecido dos Santos. Gama - DF. Brasil.

*Autor para correspondência, E-mail: ggbowen@hotmail.com

Resumo. A cardiopatia congênita mais comum encontrada em cães é a persistência do ducto arterioso (PDA), representando 25 a $30 \%$ dos casos, acomete principalmente raças puras com maior frequência em fêmeas. O ducto arterioso (DA) é uma estrutura presente na vida fetal, derivada do sexto arco aórtico, ligando à aorta a artéria pulmonar. Essa estrutura auxilia na circulação fetal, já que a falta de expansão pulmonar oferece grande resistência para o fluxo sanguíneo. Ao nascer, a expansão dos pulmões junto ao aumento de $\mathrm{PO}_{2}$ alveolar e arterial estimula a constrição do músculo liso do DA, promovendo seu fechamento. O PDA ocorre quando o fechamento do ducto não acontece. Pode apresentarse da forma clássica, com desvio de esquerda para direita, ou da forma reversa com desvio da direita para esquerda. O diagnóstico é feito a partir dos sinais clínicos junto à radiografia, eletrocardiografia, ecocardiografia e a angiocardiografia. O tratamento é cirúrgico, mas como o paciente pode desenvolver insuficiência cardíaca congestiva (ICC), é necessário um tratamento clínico prévio para possíveis arritmias e edema pulmonar. Este trabalho tem como objetivo relatar um caso de PDA na espécie canina, fêmea, da raça Maltês, com um ano de idade. Que apresentava sopro de maquinaria audível na base esquerda do coração, frêmito cardíaco palpável, os exames complementares indicavam uma grave cardiomegalia e um quadro de ICC esquerda. Após tratamento clínico, o paciente passou pela correção cirúrgica com a técnica padrão de ligadura do ducto arterioso.

Palavras chave: Cardiopatia congênita, ICC, oclusão do ducto arterioso, PDA, sopro de maquinaria

\section{Persistence ductus arteriosus: Case report}

Abstract. The most common congenital heart disease found in dogs is the shunting patent ductus arteriosus (PDA), representing 25 to $30 \%$ of cases, mainly affecting purebred breeds and is more frequent in females. The ductus arteriosus (DA) is a structure present in fetal life, derived from the sixth aortic arch, connecting the pulmonary artery to the aorta. It helps in fetal circulation since lack of lung expansion offers great resistance to blood flow. At birth, the expansion of the lungs along with the alveolar and arterial PO2 increase stimulates the DA smooth muscle constriction, promoting its closure. PDA occurs when duct closure does not occur. It may be in the classic form, with the left-to-right shift, or the reverse form with the right-to-left shift. The diagnosis is made from clinical signs along with radiography, electrocardiography, echocardiography, and angiocardiography. Treatment is surgical, but as the patient may develop congestive heart failure (CHF), prior clinical treatment is required for possible arrhythmias and pulmonary edema. This paper aims to report a case of patent ductus arteriosus in a female Maltese canine, one-year-old. It had an audible machinery murmur on the left base of the heart, a palpable thrill, and complementary exams indicated severe cardiomegaly and CHF. After clinical treatment, 
the patient underwent surgical correction using the standard technique of ductus arteriosus ligation.

Keywords: Congenital heart disease, ICC, machinery murmur, occlusion of the ductus arteriosus, PDA

\section{Persistencia del conducto arterioso: Reporte de caso}

Resumen. La cardiopatía congénita más común que se encuentra en los perros es la persistencia del conducto arterioso (PDA), que representa del 25 al $30 \%$ de los casos, afecta principalmente a las razas puras y con mayor frecuencia en las hembras. El conducto arterioso (CA) es una estructura presente en la vida fetal, derivada del sexto arco aórtico, que conecta la arteria pulmonar con la aorta. Esta estructura ayuda en la circulación fetal, ya que la falta de expansión pulmonar ofrece una gran resistencia al flujo sanguíneo. Al nacer, la expansión de los pulmones junto con un aumento en la PO2 alveolar y arterial estimula la constricción del músculo liso DA, promoviendo su cierre. PDA ocurre cuando el conducto no es cerrado. Se puede presentar de la manera clásica, con desviación de izquierda a derecha, o en forma inversa con desviación de derecha a izquierda. El diagnóstico se realiza a partir de signos clínicos junto con radiografía, electrocardiografía, ecocardiografía y angiocardiografía. El tratamiento es quirúrgico, pero como el paciente puede desarrollar insuficiencia cardíaca congestiva (ICC), es necesario un tratamiento clínico previo para posibles arritmias y edema pulmonar. Este trabajo tiene como objetivo informar un caso de PDA en la especie canina, hembra, de la raza Maltesa, con un año de edad. Quien tuvo un soplo audible en la base izquierda del corazón, una emoción cardíaca palpable, los exámenes complementarios indicaron cardiomegalia severa y un cuadro de ICC izquierda. Después del tratamiento clínico, el paciente se sometió a corrección quirúrgica utilizando la técnica estándar de ligadura del conducto arterial.

Palabras clave: Cardiopatía congénita, ICC, oclusión del conducto arterioso, PDA, soplo

\section{Introdução}

$\mathrm{Na}$ fase fetal, para que o sangue seja distribuído para todo o corpo algumas estruturas auxiliares estão presentes, como o ducto arterioso. Ele é derivado do sexto arco aórtico, ligando a artéria pulmonar à aorta descendente (Canavari et al., 2015). Normalmente, a parede do ducto é composta por $98 \%$ de músculo liso e o restante são fibras elásticas (Buchanan, 2001). Após o parto, os pulmões se expandem, diminuindo a resistência vascular pulmonar (Canavari et al., 2015). Essa expansão junto ao aumento de $\mathrm{PO}_{2}$ estimula a constrição do músculo liso do ducto, promovendo seu fechamento (Engen, 2006).

Nos cães que apresentam PDA, ocorre falha na oclusão do ducto arterioso devido uma distribuição assimétrica do componente muscular, sendo a maior parte de fibras elásticas. Esse tecido elástico intercalado impede as células do músculo liso de ocluir completamente o vaso (Nelson, 2011).

A forma clássica do PDA tem um desvio da esquerda para direita (aorta-pulmonar) por causa da diminuição da resistência vascular pulmonar e o aumento do fluxo da aorta ao nascer. Caso a pressão pulmonar seja próxima ou exceda a pressão da aorta, o desvio será de fluxo bidirecional ou de direita para esquerda (pulmonar-aorta), caracterizando o PDA reverso (Canavari et al., 2015). O PDA clássico resulta em sobrecarga de volume do ventrículo esquerdo, o que pode resultar em insuficiência cardíaca congestiva (ICC) e edema pulmonar, geralmente durante o primeiro ano de vida (Buchanan, 2001). Já a forma reversa, encontrada normalmente em animais com mais de seis meses sem o tratamento causa hipoxemia grave e cianose (Goodwin \& Tilley, 2002).

Segundo Fossum (2014), a queixa mais comum em animais sintomáticos com desvio esquerdadireita é tosse e/ou dispneia devido ao edema pulmonar. No exame físico o achado mais proeminente associado ao PDA é um ruído de maquinaria audível sobre a base esquerda do coração e frêmito palpável. Já o desvio de direita-esquerda o animal pode ser assintomáticos ou apresentar intolerância ao exercício e fraqueza de membros pélvicos durante a atividade física, além da cianose principalmente nas mucosas caudais. 
Este trabalho tem como objetivo relatar um caso de persistência do ducto arterioso na espécie canina, fêmea, da raça maltes, com um ano de idade, que foi submetida ao tratamento cirúrgico onde foi realizada a ligadura do ducto persistente.

\section{Material e métodos}

Foi atendido um canino da raça Maltês, fêmea, de um ano e dois meses de idade, pesando $2 \mathrm{~kg}$. Na anamnese a tutora relatou que o animal era menor e mais magro do que os outros filhotes da mesma ninhada, apresentava claudicação de um dos membros torácicos, estava evitando exercícios e ao exercita-se apresentava tosse.

No exame físico o animal apresentava-se alerta, com mucosas normocoradas e hidratadas, linfonodos não reativos. Na ausculta cardíaca foi encontrado um sopro audível na válvula pulmonar, também era possível sentir o frêmito cardíaco. O pulso da femoral estava hipercinético. Na aferição da pressão, apresentou $97 \mathrm{mmHg}$ PAM, $127 \mathrm{mmHg}$ PAS e 90 mmHg de PAD.

Após o exame físico, o animal foi encaminhado para radiografia de tórax na posição ventro dorsal e latero-lateral esquerda (Figura 1), em que foi constatado edema pulmonar e aumento da silhueta cardíaca. Também foi realizado o ecodopplercardiograma, que demonstrou comunicação aórtica pulmonar, determinando a persistência do ducto arterioso, branda perda de competência mitral, manutenção da capacidade contrátil do ventrículo esquerdo com sinais de sobrecarga volumétrica. No hemograma e bioquímico os resultados estavam dentro dos valores de referência.

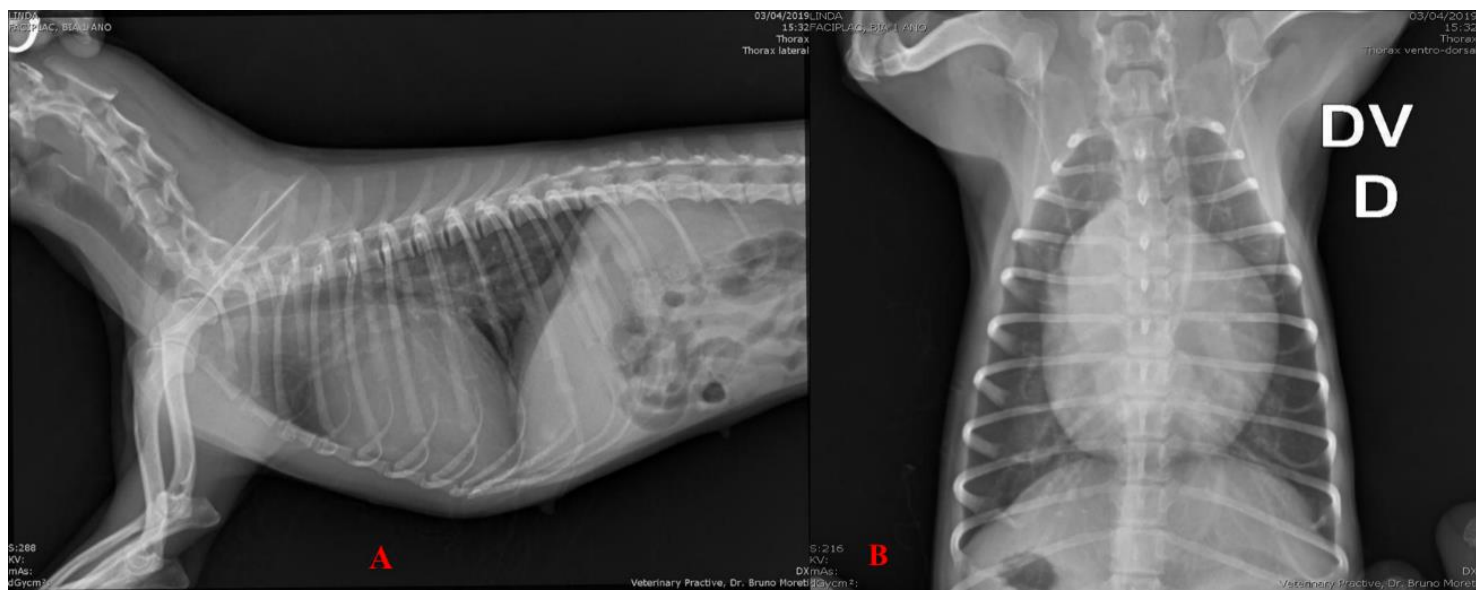

Figura 1. Projeção radiográfica de toráx ventro dorsal (A). Projeção radiográfica do toráx latero-lateral esquerda (B)

Para tratamento clínico foram utilizados furosemida e enalapril, nas doses de $1 \mathrm{mg} / \mathrm{kg}$ BID e 0,35 $\mathrm{mg} / \mathrm{kg}$ BID via oral respectivamente. Devido à queda de pressão depois de dois dias utilizando as medicações, o intervalo da furosemida passou para SID.

O animal foi encaminhado para cirurgia, a qual foi realizada no dia 25 de abril de 2019, com jejum alimentar de 12 horas e jejum hídrico de 4 horas. Por apresentar alteração sistêmica moderada, o paciente foi classificado como ASA III. Após a avaliação inicial foi administrada a medicação pré-anestésica (MPA), a qual foi composta da associação de acepromazina $(0,02 \mathrm{mg} / \mathrm{kg})$ associada a metadona $(0,2$ $\mathrm{mg} / \mathrm{kg}$ ), ambas aplicadas pela via intramuscular. A indução anestésica foi obtida pela administração intravenosa de propofol $(5 \mathrm{mg} / \mathrm{kg})$. O bloqueio intercostal foi feito com bupivacaína $(2 \mathrm{mg} / \mathrm{kg})$. Para a manutenção, realizou-se a técnica de anestesia parcial intravenosa (PIVA), administrando-se fentanil em infusão contínua $(0,3 \mathrm{mg} / \mathrm{kg} / \mathrm{h})$ concomitante ao isofluorano vaporizado em oxigênio a $100 \%$ em sistema de Baraka. Como terapia de suporte, infundiu-se solução de Ringer com Lactato na velocidade de $3 \mathrm{~mL} / \mathrm{kg} / \mathrm{h}$ com auxílio de bomba de infusão.

A técnica cirúrgica escolhida foi à ligadura do DA, como descrita por Fossum (2014). O animal foi posicionado em decúbito lateral direito, para que o toráx esquerdo ficasse livre para o acesso, após antissepsia com clorexidina degermante e álcool $70 \%$, foram posicionados os panos de campo aderente e de tecido. A toracotomia foi realizada no quarto espaço intercostal esquerdo utilizando bisturi para pele, para o subcutâneo e musculatura foi feita incisão com auxílio de um bisturi elétrico e tesoura de 
Metzenbaum. Após acessar a cavidade torácica, pulmão foi retraído caudalmente com gaze estéril umedecida, realizada incisão no pericárdio e identificou-se o nervo vago e frênico esquerdo, os mesmos foram isolados utilizando um fio de seda 2-0, já no ducto arterioso foi dissecado de maneira romba com o auxílio de uma pinça de Mixter e swabs estéreis, demonstrada na Figura 3. Ainda, com o auxílio da pinça de Mixter os fios de seda número 0 foram puxados lentamente abaixo do ducto para que fosse feita uma ligadura dupla, como pode ser visto na Figura 2.

Para a síntese torácica foram posicionados três pontos de sutura no padrão de Sultan com fio de náilon monofilamentoso $\mathrm{n}^{\circ}$ 2-0, depois os nós foram atados. Após, foi procedida a sutura de musculatura intercostal com pontos simples contínuos com o mesmo fio. Previamente ao último ponto dessa camada, foi realizada a insuflação pulmonar e então o nó foi confeccionado, manobra essa para reduzir o pneumotórax residual. A aproximação da musculatura adjacente (serrátil ventral e escaleno) e tela subcutânea foram feitas com fio de poliglactina $910 \mathrm{n}^{\circ}$ 3-0 com sutura simples contínua. Após essa etapa, um dispositivo intravenoso do tipo Scalp ${ }^{\circ} 23 \mathrm{G}$ acoplado em torneira de três vias e seringa de $10 \mathrm{~mL}$ foi disposto no oitavo espaço intercostal, conforme Figura 3, a fim de drenar o ar e restituir a pressão torácica negativa. Para a dermorrafia, foram posicionados pontos do tipo Sultan com fio de náilon monofilamentoso n 3-0.

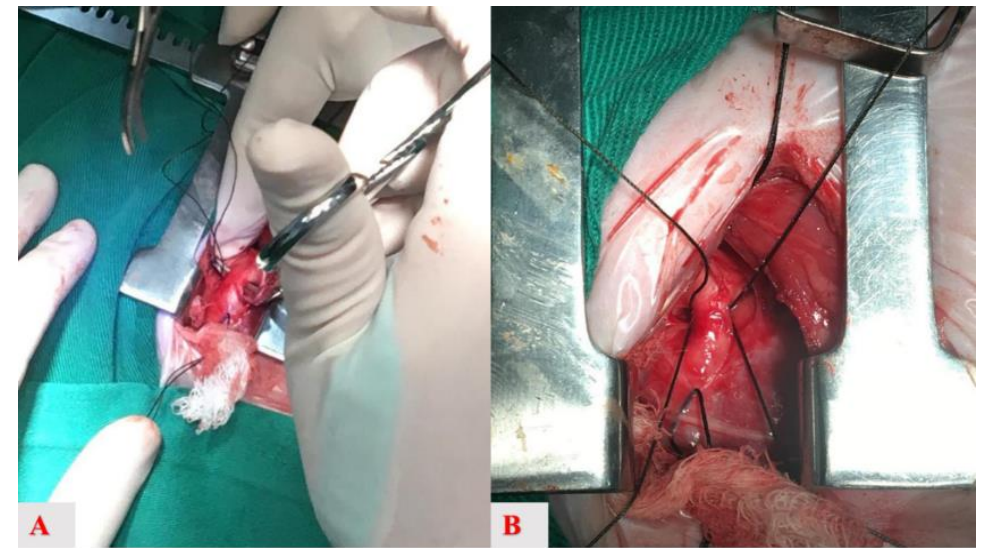

Figura 2. Ainda com a utilização da pinça de Mixter para passar os fios abaixo do ducto arterioso (A). Fios posicionados abaixo do ducto para realização da dupla ligadura $(\mathbf{B})$.

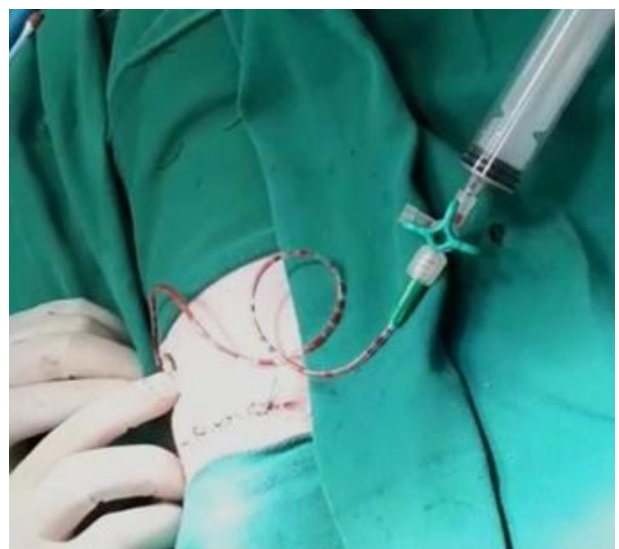

Figura 3. Restituição da pressão torácica negativa.

Logo após o fim do procedimento cirúrgico, a paciente apresentou um quadro de hipertensão, recebendo uma dose única de furosemida e foi monitorada por 24 horas. Os parâmetros avaliados foram frequência cardíaca e respiratória, pressão arterial sistólica, diastólica e média e temperatura corporal, no entanto, sem alterações.

Para o manejo pós-operatório, foi instituído analgesia com associação deTramadol e dipirona nas doses de $4 \mathrm{mg} / \mathrm{kg}$ TID e $25 \mathrm{mg} / \mathrm{kg}$ BID, respectivamente, durante 5 dias. Como antibiótico terapia foi utilizado cefalexina na dose de $30 \mathrm{mg} / \mathrm{kg}$ BID, durante 10 dias. O meloxicam foi utilizado para ação anti-inflamatória, na dose de $0,1 \mathrm{mg} / \mathrm{kg}$ SID, durante 3 dias. Foi indicada a utilização de roupa cirúrgica e curativo da ferida cirúrgica com auxílio de solução fisiológica $\mathrm{NaCl} 0,9 \%$, clorexidina e rifocina, além de colocação de bandagem compressiva, uma vez ao dia até a retirada dos pontos, que foi feita após 15 dias do procedimento.

\section{Resultados e discussão}

A cardiopatia congênita mais comum encontrada em cães é a persistência do ducto arterioso (PDA), representando 25 a $30 \%$ dos casos. Acometem principalmente os cães de raças puras como Maltês, Poodle Miniatura e Toy, Pomeranians, Yorkshire Terriers, entre outras raças e tem maior frequência em fêmeas (Fossum, 2014; Nelson, 2011). O animal relatado neste trabalho, uma fêmea da raça Maltês, se encaixa nas características descritas pelos autores acima. Além disso, apresentava um ano de idade quando foi diagnosticada com PDA, saindo da estimativa de óbito citada por Belerenian et al. (2001) que diz que $65 \%$ dos animais diagnosticados com PDA que não passam por correção cirúrgica antes de um ano de vida chegam a óbito. 
O animal apresentava tosse e intolerância ao exercício, no exame físico os achados mais proeminentes foram um ruído de maquinaria audível sobre a base esquerda do coração, frêmito palpável. O sopro contínuo de "maquinaria" auscultado na base esquerda do coração, sobrepondo-se aos sons normais, é um achado patognomônico da PDA (Sisson et al., 2004). Ele é mais alto na sístole e sua intensidade diminui gradativamente durante a diástole (Goodwin \& Tilley, 2002). No presente caso também foi encontrado o pulso da femoral hipercinético, que se dá devido à pressão sistólica estar elevada e pela baixa pressão diastólica causada pelo desvio de sangue através do ducto, quanto maior o ducto mais proeminente é o pulso (Belerenian et al., 2001; Slatter, 2003; Strickland, 2008).

Goodwin \& Tilley (2002) classificaram as pressões sanguíneas estabelecendo parâmetros para avaliação clínica, a pressão arterial sistólica (PAS) normal entre 110 a $120 \mathrm{mmHg}$ e pressão arterial diastólica (PAD) normal entre 70 a $80 \mathrm{mmHg}$. Constando-se assim, que a paciente relatada apresentava normotenso.

Os sinais radiográficos encontrados em um animal que apresenta PDA variam de acordo com o volume desviado pelo ducto arterioso, idade do animal e o grau de descompensação cardíaca (Goodwin \& Tilley, 2002). Na projeção latero-lateral, é possível visualizar o aumento do átrio, ventrículo esquerdo e edema pulmonar se houver insuficiência cardíaca congestiva (Belerenian et al., 2001). Como na imagem radiográfica da Figura 1(A), foi possível ver o edema pulmonar, constatou-se que o animal já estava no quadro de insuficiência cardíaca congestiva. O edema se dá pelos mecanismos compensatórios que são ativados na ICC, como o sistema renina angiotensina aldosterona (SRAA), que quando age de forma crônica faz a retenção de água e sódio e ocasiona não só o edema pulmonar, como também edema de membros, ascite e efusão pleural.

No exame ecodopplercardiograma realizado na paciente, foi fechado o diagnóstico de PDA e visualizado branda perda de competência da mitral. Segundo Canavari et al. (2015), o exame com Doppler colorido auxilia na localização do PDA, pois demonstra o fluxo turbulento dentro da artéria pulmonar, também é possível visibilizar um pequeno fluxo de regurgitação mitral quando há insuficiência da válvula (Belerenian et al., 2001; Canavari et al., 2015).

A farmacoterapia, com inibidores de prostaglandinas, é utilizado em humanos como o tratamento clínico para o PDA, mas não apresenta eficácia em cães e gatos, pois as características histológicas da lesão são diferentes, dado a ausência ou pouca quantidade de músculo liso na parede do ducto (Belerenian et al., 2001; Manubens, 2009; Sisson et al., 2004). Dessa forma, os animais diagnosticados com PDA clássica devem passar pelo tratamento cirúrgico. O tratamento clínico só é feito para tratar a insuficiência cardíaca congestiva esquerda, com o intuito de diminuir principalmente o edema pulmonar para que possa ser feita a cirurgia (Belerenian et al., 2001).

Para iniciar o tratamento clínico dos animais que já evoluíram para ICC esquerda, é indicado o uso da furosemida (diurético de alça) para diminuir edema pulmonar antes de o animal ser encaminhado para cirurgia (Belerenian et al., 2001). Aliado a isso, também pode ser prescritos inibidores de enzima conversora de angiotensina (ECA) que causa vasodilatação além de diminuir a retenção de sódio e água, e inotrópicos positivos que aumenta a velocidade e força de contração do miocárdio (Canavari et al., 2015; Ettinger et al., 2017; Goodwin \& Tilley, 2002). Entretanto, a administração de diuréticos e vasodilatadores deve ser feita de forma cuidadosa, porque o uso de forma demasiada pode causar hipotensão (Birchard \& Sherding, 2008; Fossum, 2014), fato este que foi observado no presente relato. O intervalo da furosemida foi alterado e não houve mais quadros de hipotensão.

Após o tratamento clínico e o controle das arritmias e edema pulmonar, o animal pode ser encaminhado para o tratamento cirúrgico. São várias as técnicas que possibilitam a oclusão do ducto arterioso. Umas delas são os stents em espiral e outros tipos dispositivos de oclusão que têm sido utilizados constantemente, com vantagem de ser um procedimento menos invasivo por não precisar da toracotomia. Realizada através do acesso da artéria femoral, a espiral é colocada no ducto com o auxílio da fluoroscopia, verificando se há oclusão completa através da injeção de contraste dentro da aorta (Fossum, 2014). Mas para que essa técnica seja realizada, deve-se ter à disposição equipamentos específicos para realizar a fluoroscopia, os animais devem pesar no mínimo $2,5 \mathrm{~kg}$ para possibilitar o acesso vascular, o tempo de duração do procedimento é maior e é ineficiente quando o ducto arterioso é grande (Johnson, 2007; Strickland, 2008). Como o paciente não apresentava o peso ideal, e o 
estabelecimento onde foi realizada a cirurgia não apresentavam os equipamentos necessários para a fluoroscopia, essa técnica foi descartada pela equipe cirúrgica.

Já a ligadura do ducto pode ser feita pela técnica padrão, técnica Jackson e toracoscopia. A técnica padrão é feita por toracotomia e dissecação do ducto arterioso, apresentando como desvantagem o risco de ruptura do ducto, enquanto a técnica de Jackson evita a dissecação do canal, pois a ligadura é feita através da região dorsal e medial da aorta, entretanto, há possibilidade de ocorrer fluxo residual (Canavari et al., 2015; Gordon \& Miller, 2005). Através da toracoscopia, o processo envolve o acesso pelo tórax e visualização através de um endoscópio e oclusão do ducto através de um clipe de titânio (Freeman, 1998). A técnica de eleição realizada neste relato foi à ligadura do ducto arterioso padrão, a dissecação foi dificultosa, devido à fibrose formada em volta do ducto que pode ter acontecido pelo diagnosticado tardio da doença, além disso, no momento da ligadura do ducto o paciente apresentou bradicardia que segundo Fossum (2014) e Stanley et al. (2003), no momento da ligadura pode ocorrer uma bradicardia reflexa devido ao aumento súbito na pressão aórtica.

Se o diagnóstico for precoce e o paciente não apresentar uma cardiomegalia grave, no pós-cirúrgico podem apresentar-se clinicamente normais e o tamanho do coração tende a se normalizar. Se a válvula mitral for estruturalmente normal, a regurgitação mitral e o consequente sopro sistólico se resolvem após a ligadura ou oclusão do ducto (Eyster, 1998; Ware, 2006). Logo após a cirurgia o paciente relatado apresentava ausculta cardíaca normal, sem o ruído de maquinaria e frêmito cardíaco palpável. Um mês após a cirurgia foi realizado radiografia, demonstrada na Figura 4, onde pode ser visto uma diminuição significativa na silhueta cardíaca.

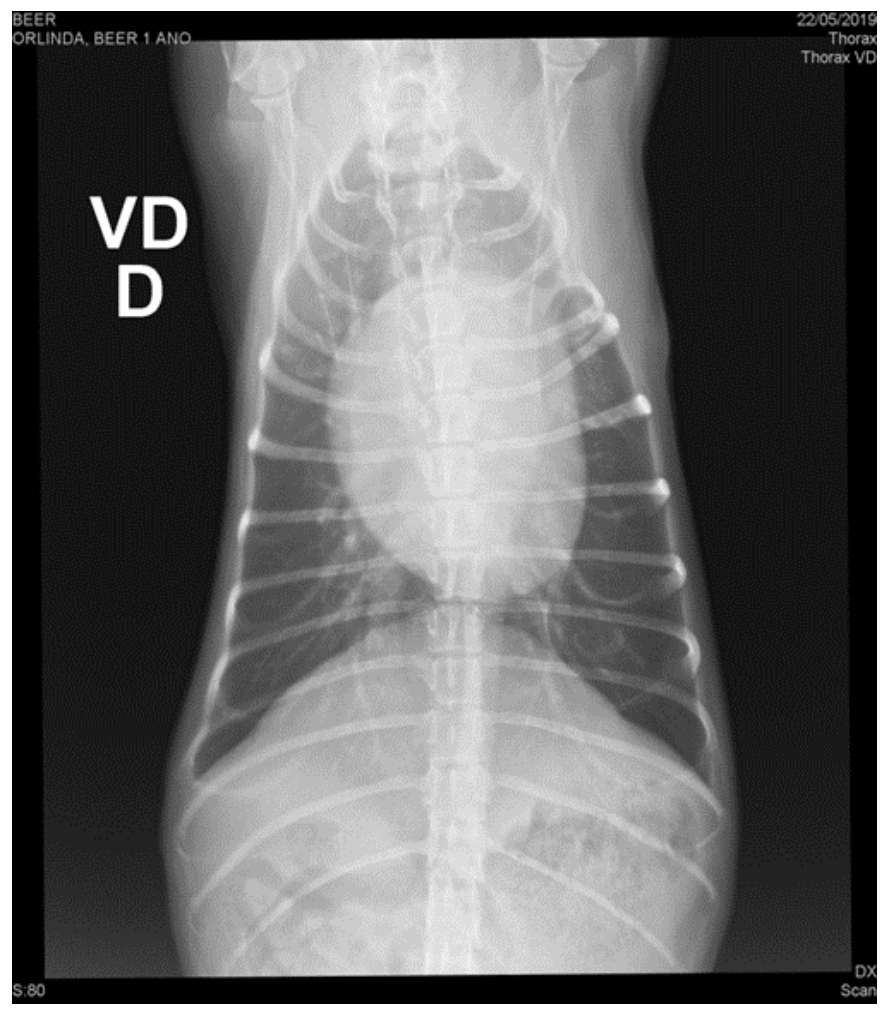

Figura 4. Radiografia torácica um mês após cirurgia.

A ecocardiografia não indicou fluxo residual no ducto arterioso, mas indicou leve refluxo na mitral, que segundo Ishikawa et al. (2013), acontece quando a sobrecarga de volume nas câmaras cardíacas ocorre por um período prolongado.

Para anestesia, o animal foi classificado em ASA IV, que de acordo com American Society of Anesthesiologits (2019) é aplicado em pacientes com doença sistêmica grave que ameaça a vida. A acepromazina, fármaco usado na MPA, é um derivado de fenotiazínico, com capacidade de produzir sedação e relaxamento muscular. No sistema cardiovascular seus efeitos são complexos, agindo diretamente sobre o coração (promovendo efeitos antiarrítmicos) e os vasos sanguíneos (causando 
vasodilatação por bloqueio dos receptores alfa-1 e dopaminérgicos), podendo causar hipotensão (Spinosa et al., 2017). Como a paciente encontrava-se normotensa no pré-cirúrgico de acordo com a classificaçao proposta por Lima (2017), em que a hipertensão se estabelece com PAS > $180 \mathrm{mmHg}$ ou $\mathrm{PAM}>140 \mathrm{mmHg}$ o fármaco pode ser utilizado com segurança.

Por se tratar de uma toracotomia intercostal, cirurgia considerada de alto potencial álgico (Resende et al., 2014), alguns dos opioides indicados para a analgesia são a morfina, fentanil, hidromorfona, oximorfona e metadona (Drobatz et al., 2019). No caso relatado, optou-se pela administração de metadona na MPA com infusão contínua de fentanil no trans-operatório, pois eram os fármacos disponíveis no estabelecimento veterinário. Para a indução anestésica optou-se pela administração de propofol pela via intravenosa, que apresenta efeito cardioprotetor (Ansley et al., 2016).

Dentre os fármacos anestésicos inalatórios, cita-se a utilização de halotano, isofluorano e sevofluorano na manutenção anestésica de pacientes submetidos à toracotomia. Dentre os fármacos supracitados, o sevofluorano e o isofluorano são os menos arritmogênico, no entanto, o isofluorano é o medicamento de mais fácil acesso ao médico veterinário e que apresenta baixa metabolização hepática (Grimm et al., 2015). Somado a isso, por conta da terapia antálgica preventiva utilizada (metadona, fentanil e bupivacaína), a CAM do isofluorano pode ser diminuída, fazendo com que o paciente receba menores concentrações de isofluorano.

Para o bloqueio intercostal foi utilizado a bupivacaína, que é cerca de quatro vezes mais potente que a lidocaína, com início lento de ação (20 a 30 min) e longa duração do efeito ( 3 a 10 h) (Grimm et al., 2015). O bloqueio local é feito com o intuito de auxiliar no controle da dor no pós-operatório, diminuindo também o uso dos analgésicos.

\section{Conclusão}

É fundamental que os animais, principalmente as raças de maior risco, passem pelos exames necessários para diagnosticar o PDA ainda nos primeiros meses de vida. O diagnóstico de forma precoce evita a insuficiência cardíaca congestiva e o remodelamento cardíaco, desta forma o paciente pode passar pela correção cirúrgica e viver sem sequelas.

\section{Referências bibliográficas}

Ansley, D. M., Raedschelders, K., Choi, P. T., Wang, B., Cook, R. C., \& Chen, D. D. Y. (2016). Propofol cardioprotection for on-pump aortocoronary bypass surgery in patients with type 2 diabetes mellitus (PRO-TECT II): a phase 2 randomized-controlled trial. Canadian Journal of Anesthesia/Journal Canadien d'anesthésie, 63(4), 442-453. https://doi.org/10.1007/s12630-015-0580-z

Belerenian, G. C., Camacho, A. A., \& Mucha, C. J. (2001). Afecciones cardiovasculares en pequeños animales. Ed. Inter-Médica.

Birchard, S. J., \& Sherding, R. G. (2008). Manual Saunders: clínica de pequenos animais. In Ed. Roca (Vol. 3).

Buchanan, J. W. (2001). Patent ductus arteriousus morphology, pathogenesis, types and treatment. Journal of Veterinary Cardiology, 3(1), 7-16.

Canavari, I. C., Kunz, F. A., Pereira, E. Z., \& Costa, M. T. (2015). Abordagem clínica da persistência do ducto arterioso em cães: revisão de literatura. Revista Científica de Medicina Veterinária, 1-16.

Drobatz, K. J., Hopper, K., Rozanski, E. A., \& Silverstein, D. C. (2019). Textbook of Small Animal Emergency Medicine. John Wiley \& Sons.

Engen, R. L. (2006). Dinâmica do sistema cardiovascular. In: DUKES, H. H. Fisiologia Dos Animais Domésticos. 12. Ed. Rio de Janeiro: Guanabara Koogan, Cap. 10, 12, 163-172.

Ettinger, S. J., Feldman, E. C., \& Cote, E. (2017). Textbook of Veterinary Internal Medicine-eBook. Elsevier Health Sciences.

Eyster, G. E. (1998). Procedimentos Cirúrgicos Cardíacos Básicos. Manual de Cirurgia de Pequenos Animais, 2, 1075-1104.

Fossum, T. W. (2014). Cirurgia de pequenos animais (4th ed., Vol. 1). Elsevier Brasil. 
Freeman, L. J. Introduction. In: Freeman, L. J. Veterinary Endosurgery. St Louis: Mosby, 1998. Cap. 1, p. 3-23.

Goodwin, J. K., \& Tilley, L. P. (2002). Manual de Cardiologia para Cães e Gatos. São Paulo: Editora Roca, Cap, 14, 259-267.

Gordon, S. G., \& Miller, M. W. (2005). Transarterial Coil Embolization for Canine Patent Ductus Arteriosus Occlusion. Clinical Techniques in Small Animal Practice, 20(3), 196-202. https://doi.org/10.1053/j.ctsap.2005.05.008

Grimm, K., Lamont, L., Tranquilli, W., Greene, S., \& Robertson, S. (2015). Anestesiologia e analgesia em veterinária. Editora Roca.

Ishikawa, R., Fujii, Y., Takano, H., Sunahara, H., Aoki, T., \& Wakao, Y. (2013). Left Ventricular Reverse Remodeling After Ductal Closure in Dogs with Hemodynamically Significant Patent Ductus Arteriosus. Journal of Applied Research in Veterinary Medicine, 11, 66-69.

Johnson, M. S. (2007). Options for treatment of patent ductus arteriosus in dogs. Companion Animal, 12(1), 43-45. https://doi.org/10.1111/j.2044-3862.2007.tb00114.x

Lima, M. P. A. (2017). Monitoração do paciente crítico. In: Cadernos Técnicos de Veterinária e Zootecnia: Emergência Em Medicina Veterinária. 87. Ed. Belo Horizonte: Conselho Regional de Medicina Veterinária do Estado de Minas Gerais - CRMV-MG, 87, 56-69.

Manubens, J. (2009). Patent ductus arteriosus: clinical management. Proceedings of the Southern European Veterinary Conference \& Congreso Nacional Da Asociación de Veterinarios Españoles Especialistas En Pequeños Animales, Barcelona.

Nelson, R. (2011). Medicina interna de pequenos animais. Elsevier Brasil.

Resende, F. A., Mourão, M. B. A., Allevato, B. S. C., Oliveira, B. A. M. F. M., Oliveira, C. H. S., \& Silva, S. R. B. (2014). Analgesia para toracotomia com cateter multiperfurado e bomba elastomérica: relato de caso. Rev Med Minas Gerais, 24(Supl 3), S68-S72.

Sisson, D. D., Thomas, W. P., \& Bonagura, J. D. (2004). Cardiopatia congênita. Tratado de Medicina Interna Veterinária: Doenças do Cão e do Gato, 1, 788-817.

Slatter, D. H. (2003). Textbook of small animal surgery (Vol. 1). Elsevier Health Sciences.

Spinosa, H. S., Górniak, S. L., \& Bernardi, M. M. (2017). Farmacologia aplicada à medicina veterinária. Grupo Gen-Guanabara Koogan.

Stanley, B. J., Luis-Fuentes, V., \& Darke, P. G. G. (2003). Comparison of the incidence of residual shunting between two surgical techniques used for ligation of patent ductus arteriosus in the dog. Veterinary Surgery, 32(3), ajvet50025. https://doi.org/10.1053/jvet.2003.50025

Strickland, K. N. (2008). Pathophysiology and therapy of heart failure. In Manual of canine and feline cardiology (pp. 288-314). Elsevier.

Ware, W. A. (2006). Anomalias cardíacas congênitas comuns. NELSON, RW; COUTO, CG Medicina Interna de Pequenos Animais. 3ed. São Paulo: Elsevier, 147-164.

\section{Histórico do artigo:}

Recebido: 23 de junho, 2020 .

Aprovado: 2 de agosto, 2020.

Disponível online: 30 de outubro, 2020.
Licenciamento: Este artigoé publicado na modalidade Acesso Aberto sob a licença Creative Commons Atribuição 4.0 (CC-BY 4.0), a qual permite uso irrestrito, distribuição, reprodução em qualquer meio, desde que $\mathrm{o}$ autor e a fonte sejam devidamente creditados. 